\title{
Evaluating the Dark Matter Contribution to Galactic Synchrotron Radiation
}

\section{Tim Linden ${ }^{* \dagger}$}

Department of Physics, University of California, 1156 High Street., Santa Cruz, CA 95064

E-mail: tlinden@ucsc.edu

\section{Stefano Profumo}

Department of Physics, University of California, 1156 High Street., Santa Cruz, CA 95064 Santa Cruz Institute for Particle Physics, Santa Cruz, CA 95064

E-mail: profumo@scipp.ucsc.edu

\section{Brandon Anderson}

Department of Physics, University of California, 1156 High Street., Santa Cruz, CA 95064

E-mail: anderson@physics.ucsc.edu

\begin{abstract}
Currently, a significant effort is underway to observe indirect evidence of dark matter annihilation in our galaxy. One interesting finding was an unexpected synchrotron haze (the "WMAP haze") with a similar intensity and morphology to those predicted by dark matter models. This might also be connected to another recent puzzle in cosmic ray physics: the excess of high energy positrons reported by the Pamela satellite. We create models of the synchrotron component expected from dark matter annihilation and compare these with the observed WMAP excesses. We further analyze the expected Fermi signal stemming from these models, and place constraints on the magnetic fields necessary to match these multi-wavelength observations.
\end{abstract}

Identification of Dark Matter 2010-IDM2010

July 26-30, 2010

Montpellier France

* Speaker.

${ }^{\dagger}$ A footnote may follow. 


\section{Introduction}

A long discussed explanation for the observed abundance of dark matter is the addition of a stable supersymmetric particle which contributes to the energy density of the universe without interacting electromagnetically [1]. If this particle additionally interacts with baryonic matter through the weak force, then the correct dark matter relic density falls out naturally, and the particle is commonly termed weakly interacting massive particle (WIMP) [2]. WIMP particles with masses on the order of $1 \mathrm{TeV}$ and velocity dependent cross-sections on the order of $10^{-26} \mathrm{~cm}^{3} \mathrm{~s}^{-1}$ are potentially detectable both directly through their collisions with baryonic matter on Earth as well as indirectly through the stable baryons and leptons produced by WIMP annihilation [3].

WIMPs, with masses consistent with the weak scale, will annihilate to produce stable leptons in the range of 1-100 GeV. These stable leptons will, in turn, produce synchrotron radiation as they propagate through the galactic magnetic field. If these magnetic fields are on the order of $\sim 10 \mu \mathrm{G}$ [4], then this synchrotron emission should peak in the 10-100 Ghz band. In fact, a significant excess emission located symmetrically around the galactic center was first discovered by $[5,6]$ and dubbed the "WMAP Haze". The spectrum and morphology of this signal make it to explain with standard astrophysical emission processes. This makes the WMAP haze an interesting test for theories of dark matter annihilation, as the intensity and spectrum are well modeled by standard dark matter annihilation scenarios [7, 8].

In this study, we examine the dark matter matches to the WMAP haze proposed by [7, 8], noting that the morphology of the dark matter synchrotron signal (hereafter, the dark matter haze), is sensitively dependent not only on the details of the dark matter annihilation pathway, but also on the details of cosmic ray propagation in the inner galaxy. We find that when cosmic ray propagation is constrained to agree with local observations of the cosmic ray primary to secondary ratios, the morphology of dark matter synchrotron radiation falls off much faster as a function of latitude than the observed WMAP haze. We then evaluate how this signal may be influenced by uncertainties in the cosmic-ray propagation parameters, and finally discuss how higher energy experiments such as Fermi may constrain these scenarios. These proceedings are based on Ref. [9], to which the reader is referred for further details.

\section{Simulation Models}

The parameter space of WIMP models is formidable in size, and thus a complete analysis of their annihilation pathways is beyond the scope of this study. Instead, we focus on three models which we expect to create bounds on the lepton spectrum produced by dark matter annihilations. First, we consider a model of a $40 \mathrm{GeV}$ WIMP annihilating to $b \bar{b}$, a model which we dub Soft to describe the lepton annihilation spectrum. Secondly, we employ a model of a $1.5 \mathrm{TeV}$ WIMP annihilating to $\mu^{+} \mu^{-}$, which we dub Hard. Lastly, we consider an intermediate case of a $200 \mathrm{GeV}$ WIMP annihilating to $W^{+} W^{-}$, in agreement with the model proposed by [10] to resolve the PAMELA anomaly. In Figure 1 (left), we show the spectrum of stable leptons produced via each pathway.

In order to calculate the expected synchrotron flux due to leptons generated by WIMP annihilation, we have employed the Galprop cosmic ray propagation code [11, 12], which has 

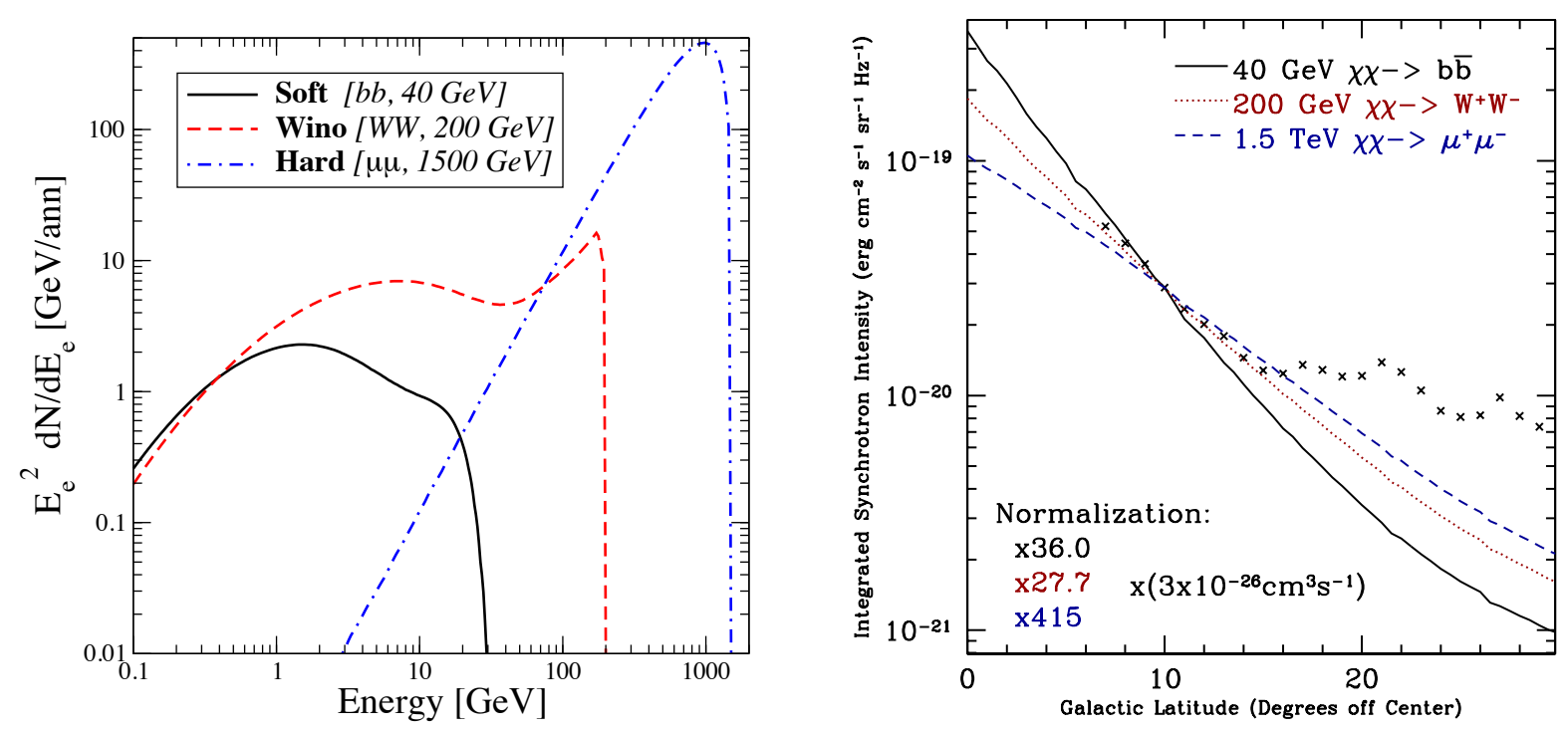

Figure 1: Left: The spectrum (multiplied by energy squared) of electrons (and positrons) produced by the pair annihilation of dark matter for our three benchmark models: Soft (featuring a lightest neutralino mass of $m_{\chi}=40 \mathrm{GeV}$ and a dominant annihilation final state into $\left.\bar{b} b\right)$, Wino $\left(m_{\chi}=200 \mathrm{GeV}, W^{+} W^{-}\right)$and Hard $\left(m_{\chi}=1.5 \mathrm{TeV}, \mu^{+} \mu^{-}\right)$. Right: Simulated dark matter haze for three dark matter channels $40 \mathrm{GeV} \chi \chi->$ $b \bar{b}$ (Soft, black solid), $200 \mathrm{GeV} \chi \chi->W^{+} W^{-}$(Wino, red dotted), $1500 \mathrm{GeV} \chi \chi->\mu^{+} \mu^{-}$(Hard, blue dashed)) plotted against the WMAP haze as determined by [8].

been designed to solve the cosmic ray transport equation, taking into account effects such as diffusion, re-acceleration and convection, as well as energy loss mechanisms due to synchrotron, bremsstrahlung, and inverse Compton scattering. To obtain accurate estimates of the additional lepton flux due to dark matter annihilation, we have customized the Galprop code to accept an input lepton flux created by DarkSUSY [13], which employs particle physics models to determine the pathway and mass dependent flux of leptons from supersymmetric dark matter candidates.

A wide variety of input parameters (e.g. the diffusion coefficient, Alfvén velocity, convection velocity, and the galactic magnetic and radiation fields) must be correctly chosen in order to ensure that the Galprop output provides a reasonable match for galactic synchrotron emission. However, not all of these input parameters are well constrained theoretically - thus it is important to ensure that any Galprop simulation provides reasonable outputs by comparing the primary to secondary ratio observed at the solar position to that determined by the cosmic ray probes such as HEAO3 [14], CRIS [15], and ISOMAX [16]. We pick a default model including a diffusion constant $\mathrm{D}_{0}=5 \times 10^{28} \mathrm{~cm}^{2} \mathrm{~s}^{-1}$, an Alfvén velocity $\mathrm{v}_{\alpha}=25 \mathrm{~km} \mathrm{~s}^{-1}$, a simulation height of $4 \mathrm{kpc}$ and negligible convection, which provides a reasonable match to these cosmic ray constraints.

\section{Results}

We find that the synchrotron signal from dark matter annihilation has a morphology which decays much more quickly as a function of latitude than the observed WMAP haze. In Figure 1 (right), we show the simulated WMAP haze for our baseline model, compared to the WMAP 


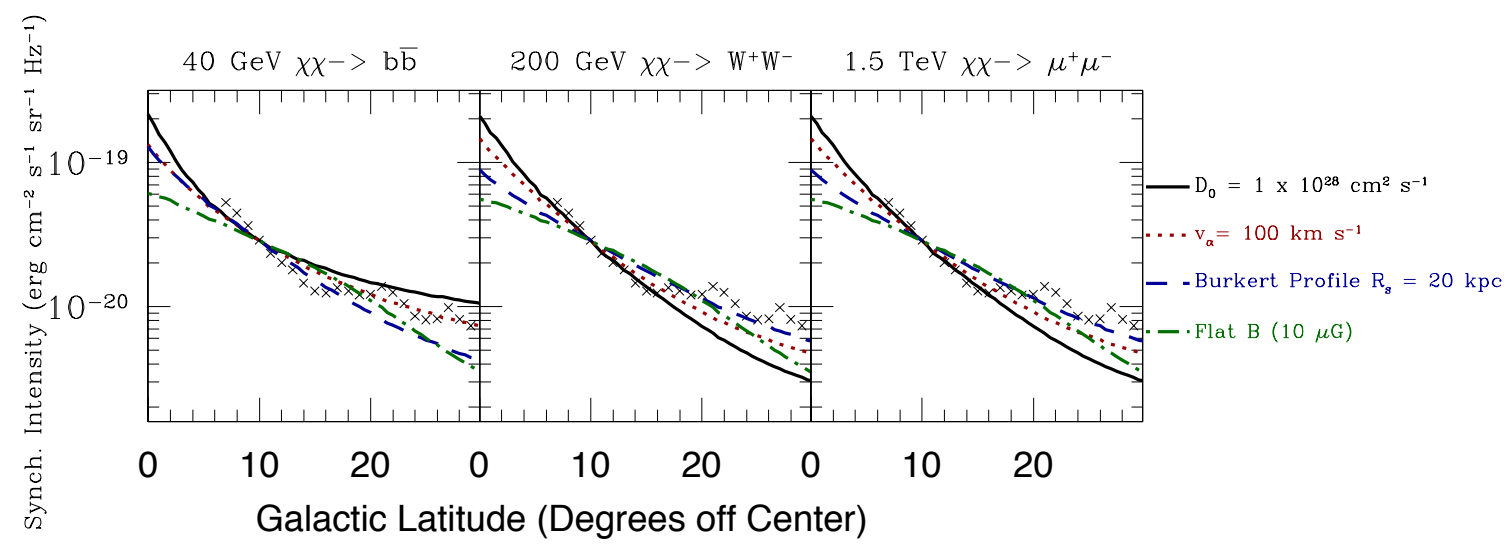

Figure 2: Simulated dark matter haze for three dark matter channels (40 GeV $\chi \chi->b \bar{b}$ (Soft, left), 200 $\mathrm{GeV} \chi \chi->W^{+} W^{-}$(Wino, center), $1500 \mathrm{GeV} \chi \chi->\mu^{+} \mu^{-}$(Hard, right)) for illustrative choices in both propagation parameters and dark matter profiles which provide a good synchrotron match to the observed WMAP haze, including: a diffusion constant $D_{0}=1 \times 10^{28} \mathrm{~cm}^{2} \mathrm{~s}^{-1}$ (black solid), an Alfvén velocity of $v_{\alpha}=100 \mathrm{~km} \mathrm{~s}^{-1}$ (red dotted), a dark matter distribution following a Burkert profile with scaling radius $R_{s}=11.6 \mathrm{kpc}$ (blue dashed), and a flat magnetic field of strength $10 \mu \mathrm{G}$ (green dash-dot).

haze calculated by [8] using the haze subtraction template CMB5. Because effects such as substructure or Sommerfeld enhancement may alter the effective dark matter cross-section from the value implied by the thermal relic density, we have allowed the intensity of the synchrotron signal to float from the base value of $3 \times 10^{26} \mathrm{~cm}^{3} \mathrm{~s}^{-1}$ in order to match the intensity of the observed WMAP haze at $10^{\circ}$ below the galactic center. In Figure 1 we have noted the multiplicative constants necessary to fix this intensity for each model.

We note that the parameters controlling the propagation of leptons from the inner galaxy, where most WIMP annihilations are expected, into the intermediate latitudes, where the WMAP haze is observed, are highly uncertain. The most important parameters which affect the synchrotron distribution are the diffusion constant and Alfvén velocity, which control the diffusion through position and momentum space respectively. We find that substantial changes in either of these parameters may allow for a dark matter fit to the WMAP haze. By evaluating changes in each parameter independently, we find good fits for the WMAP haze using either a lower diffusion constant of $\mathrm{D}_{0}=1 \times 10^{28} \mathrm{~cm}^{2} \mathrm{~s}^{-1}$ or an Alfvén velocity $\mathrm{v}_{\alpha}=100 \mathrm{~km} \mathrm{~s}^{-1}$. In Figure 2 we show these fits (black solid and red dotted respectively) compared to the observed WMAP haze. Alternative changes, such as exceptionally large scale heights (in excess of $16 \mathrm{kpc}$ ), do not allow dark matter to replicate the morphology of the WMAP haze.

However, such severe alterations in these parameters will also influence the observed primary to secondary ratio at the solar position. In Figure 3 we model the parameter space of simultaneous changes in the diffusion constant and Alfvèn velocity, as well as the diffusion constant and the height of the diffusion region - using a $\chi^{2}$ fit to compare the simulated primary to secondary ratio to observations $[14,15,16]$. We find that corresponding changes in the diffusion constant and Alfvèn velocity are highly constrained to the central values adopted in this study, while changes in the diffusion constant and the height of the diffusion box are constrained to lie along a line where 

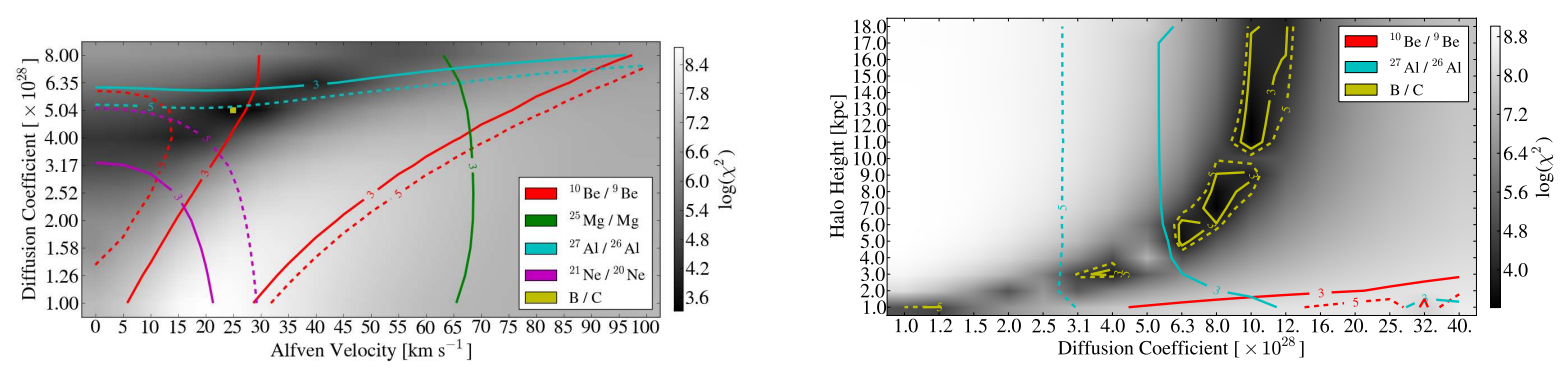

Figure 3: Left: Constraints from Nuclei fluxes and primary to secondary ratios as a function of Alfvén velocity and diffusion constant. Darker areas indicate better fits to simulation data (lower $\left.\chi^{2}\right)$. The minimum falls around a diffusion constant of $5.0 \times 10^{28} \mathrm{~cm}^{2} \mathrm{~s}^{-1}$ and an Alfv? en velocity of $25 \mathrm{~km} \mathrm{~s}^{-1}$. Right: Same as left for changes in the diffusion constant and simulation height. A degeneracy exists along a line of constant diffusion constant divided by height. Colored lines for each ratio show 3 and 5 sigma constraints on the dataset.

their ratio is held constant. From these constraints, we find no models where changes in the cosmic ray propagation setup can create dark matter models which correctly match the WMAP haze.

While substantial efforts have been undertaken to determine the large scale structure of dark matter in the Milky Way [17, 18], small deviations are still possible - especially with regards to the effect of dense baryons on the dark matter structure in the inner galaxy [19]. Since the annihilation rate of dark matter depends on the square of the dark matter density - we find the lepton injection spectrum to be consistent for the NFW, Via Lactea, and Einasto profiles - so long as the crosssection is allowed to float in order to match the WMAP intensity for each model. However, in the case of the Burkert profile - the annihilation rate near the galactic center is sub-dominant to annihilations at higher radii. In Figure 2 (blue dashed), we show that this smooths out the latitude dependence of the dark matter haze, providing a reasonable match to WMAP observations. We note that the enhanced cross-sections necessary for the Burkert model may make the signal incompatible with multi-wavelength observations. Furthermore, this constraint would also apply to any other method (such as substructure) which aims to increase the relative intensity of high latitude dark matter annihilations.

Finally, we note that the magnetic field models away from the solar position are highly uncertain, and local measurements of cosmic ray activity are insufficient to constrain this parameter space. Thus, in this study, we seek only to determine the extent to which changes in the magnetic field may alter the morphology of the dark matter haze. In Figure 2 (green, dot-dashed), we plot the expected synchrotron signal for a flat magnetic field distribution. We find that the expected falloff in the magnetic field is greatly diminished if the magnetic field remains constant in the region between 1-3 kpc below the galactic center, and thus provides a reasonable match to the WMAP haze.

\section{Discussion and Conclusion}

Any additional input spectrum of leptons due to dark matter annihilation will not only produce synchrotron radiation, but also $\gamma$-rays through inverse Compton scattering with photons in the interstellar medium. The relative intensity of energy in radio and $\gamma$-rays depends almost exclusively 


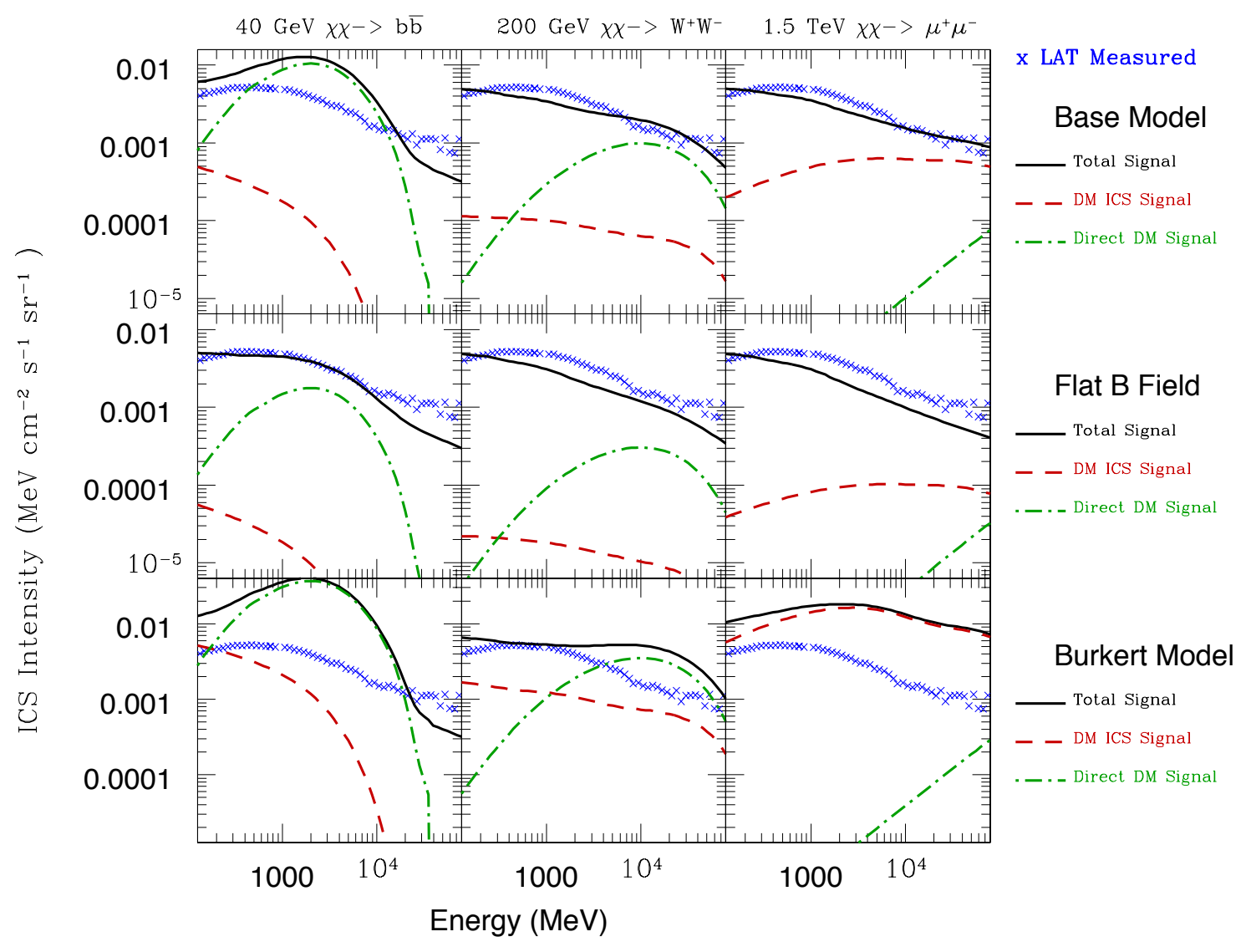

Figure 4: Expected Fermi Signal for models including the base model (top), a flat B-field of $10 \mu G$ (middle), and a model employing a Burkert profile with $R_{s c}=11.6 \mathrm{kpc}$ bottom, for each of the three dark matter decay channels (40 GeV $\chi \chi->b \bar{b}$ (Soft, left), $200 \mathrm{GeV} \chi \chi->W^{+} W^{-}$(Wino, center), $1500 \mathrm{GeV} \chi \chi->\mu^{+} \mu^{-}$ (Hard, right)). The red dash shows the expected Inverse Compton (ICS) emission based on the Galprop model for the ISRF, while the green dot-dashed line is based on the direct $\gamma$-ray production from the relevant DarkSUSY model, and normalized to the input dark matter density profile. The total emission (black solid) includes Galprop based emission for $\pi^{0}$-decay and bremsstrahlung emission, as well as power law models for the extragalactic background (Energy ${ }^{-2.45}$ normalized to $1.1 \times 10^{-3} \mathrm{MeV} \mathrm{cm}^{-2} \mathrm{~s}^{-1} \mathrm{sr}^{-1}$ at $1 \mathrm{GeV}$ ) and point sources (Energy ${ }^{-2.4}$ normalized to $3.1 \times 10^{-4} \mathrm{MeV} \mathrm{cm}^{-2} \mathrm{~s}^{-1} \mathrm{sr}^{-1}$ at $1 \mathrm{GeV}$ ). The observed Fermi-LAT $\gamma$-ray flux is obtained from [20].

on the relative energy densities of the magnetic field and photons, which is believed to be of near order unity in the WMAP haze region [21]. In Figure 4 we show the expected ICS contribution at mid-latitudes in the Fermi energy band. We find that Fermi limits on the direct photons from dark matter annihilation rule out the use of the Burkert profile in order to match the WMAP morphology. We note that this limit would apply not only to a Burkert profile, but to any profile which attempts to smooth out the WMAP morphology by creating it's leptons directly in the WMAP haze region. In the case of the base model, we find that the soft annihilation spectrum is ruled out, while the harder spectra stay within Fermi limits. Finally, alterations which allow stronger magnetic fields in the WMAP region, similar to our flat magnetic field model, allow matches to the WMAP intensity 
and spectrum without violating limits from Fermi.

In this study, we have investigated the morphology of the dark matter induced synchrotron signal and found that the WMAP haze is not naturally modeled by dark matter annihilation, so long as cosmic rays are universally constrained to have diffusion parameters such that local primary to secondary ratios are held consistent with observations. However, changes in the magnetic field intensity in the haze region remain an important uncertainty, as they may greatly change the morphology of synchrotron emission without affecting cosmic ray propagation. However, changes in this parameter will effect not only the morphology of a dark matter synchrotron signature as well as the foreground astrophysical emission signatures which must be subtracted in order to extract a WMAP haze - potentially altering the morphology or intensity of the observed WMAP haze. Thus, while a dark matter match to the WMAP remains possible due to large uncertainties in the galactic magnetic field intensities, the existence of the WMAP haze alone does not provide compelling evidence for dark matter annihilation in the inner galaxy.

\section{References}

[1] G. Bertone, D. Hooper and J. Silk Phys. Rept. 405, 279 (2005)

[2] B. W. Lee and S. Weinberg Phys. Rev. Lett. 39165 (1977)

[3] E. Aprile and S. Profumo New Journ. of Phys. 11 (2009)

[4] H. Ohno and S. Shibata MNRAS 2624 (1993)

[5] D. P. Finkbeiner, Astrophys. J. 614, 186 (2004).

[6] D. P. Finkbeiner, [arXiv:0409027 [astro-ph]]

[7] D. Hooper, G. Dobler and D. P. Finkbeiner Phys. Rev. D 768 (2007)

[8] G. Dobler, D. P. Finkbeiner, Astrophys. J. 680, 1222-1234 (2008). [arXiv:0712.1038 [astro-ph]].

[9] T. Linden, S. Profumo and B. Anderson Phys. Rev. D 826 (2010)

[10] G. Kane, R. Lu and S. Watson Physics Letters B. 681151 (2009)

[11] A. W. Strong and I. V. Moskalenko Astrophys. J. 509, 212, (1998)

[12] A. W. Strong et al., [arXiv:0907.0559 [astro-ph]]

[13] P. Gondolo et al., JCAP 0407008 (2004)

[14] J. J. Engelmann, P. Ferrando, A. Soutoul, P. Goret, E. Juliusson Astron. Astrophys. 233, 96 (1990)

[15] N. E. Yanasak et al., Astrophys. J. 563768 (2001)

[16] T. Hams et al., Astrophys. J. 611892 (2004)

[17] J. Diemand et al., Nature 454735 (2008)

[18] V. Springel et al. MNRAS 3911685 (2008)

[19] A. Burkert Astrophys. J. Lett. 447 L25+ (1995)

[20] A. A. Abdo et al. Phys. Rev. Lett 103251101 (2009)

[21] A. W. Strong, I. V. Moskalenko and O. Reimer, Astrophys. J. 537, 763 (2000), Erratum: Ibid., 541, 1109 (2000). 